Actas VII Congr. Intern. Est. Pirenaicos, 7 (1): 5 a 16

\title{
Hoque, apellatif générique de cours d'eau dans le Couserans *
}

\author{
François BaBY **
}

«Recueillir tous les appellatifs régionaux qui sont encore vivants» était l'une des tâches que, voici bientôt vingt ans, Paul LEBEL proposait à la recherche hydronymique française ${ }^{1}$.

Il semble que dans la Roamnia le mot HoQue n'ait jamais été relevé comme appellatif générique de cours $d$ 'eau: il désigne pourtant encore aujourd'hui, et dans la pratique courante du langage, quatre-vingts ruisselets du Haut-Couserans.

Une monographie exploratoire de ce terme passe nécessairement par une étude transdisciplinaire qui tienne compte des données géographiques, issues notamment de la géologie et de l'hydrologie, avant d'aboutir à un essai de définition et à des conclusions proprement linguistiques.

\section{Situation}

Aire. - Les hoques désignent, dans le canton d'Oust, arrondissement de Saint-Girons (Ariège), la plupart des affluents des ruisseaux d'Ebints, d'Estours, d'Angouls et de l'Alet, eux-mêmes affluents du Salat, et tous les menus cours d'eau qui se jettent directement dans cette rivière en amont de Seix. L'ensemble délimite une aire continue de $260 \mathrm{~km} \mathrm{2,} \mathrm{bornée} \mathrm{au}$ Sud par l'Espagne, et couvrant entièrement les communes de Seix, Couflens et Ustou ainsi qu' une infime partie de celle de Sentenac-d'Oust ${ }^{2}$. Le mot ne paraît pas connu ailleurs.

\footnotetext{
Comunicación leida en las sesiones de la Sección VI: Filología, Lingüística y Literatura pirenaicas, en Seo de Urgel, 1974.

* Agrégé des Lettres. Assistant à l'Université de Toulouse-Le Mirail.

1. LEBel, P.: Principes et néthode d'hydronymie française, Paris, Les BellesLettres, 1956, pp. 18-19.

2. Cf. carte in fine.
} 
Si l'on relève trente-huit mentions de cet appellatif sur les cartes I. G. N. au $1 / 20.000$, le cadastre de $1838-39$ en livre quatre-vingts, maintenues dans l'usage actuel, mais limitées à cinquante-huit dans les cadastres rénovés de $1956-60^{3}$. Comme pour l'ensemble de la zone pyrénéenne, CASsini ne note pas le mot ${ }^{4}$, mais les terriers anciens, de 1519 à 1778 , proposent cinquante-sept occurences supplémentaires dont vingt-quatre restent non identifiées malgré de nombreuses enquêtes sur le terrain ${ }^{5}$. Au total le mot avait, au XVIII ${ }^{\mathrm{e}}$ siècle, une densité supérieure à $1 / 2 \mathrm{~km}^{2}$; la normalisation cadastrale par «ruisseau» ou «ravin» $\mathrm{l}^{\prime}$ a faite régresser aujourd'hui à un peu moins de $1 / 3 \mathrm{~km}^{2}$, avec la répartition suivante:

\begin{tabular}{|c|c|c|c|c|c|}
\hline & Seix & Couflens & Ustou & $\begin{array}{c}\text { Sentenac } \\
\text { d'Oust }\end{array}$ & Total \\
\hline 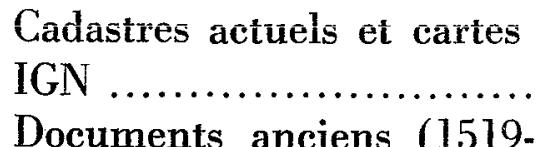 & 18 & 32 & 29 & 1 & 80 \\
\hline 1778) avec identification . & 13 & 18 & 2 & 0 & 33 \\
\hline Non identifiées ............ & 9 & 15 & 0 & 0 & 24 \\
\hline Total ... & 40 & 65 & 31 & 1 & 137 \\
\hline
\end{tabular}

\section{Caractérisation}

Les hoques sont toujours des affluents mineurs, dont la longueur varie de 600 à 3000 mètres mais dont la pente atteint de 450 à 650 mètres par kilomètre. Dans le bassin du Haut-Salat, fortement tourmenté, l'altitude de leurs sources n'est jamais inférieure à $1300 \mathrm{~m}$, mais en moyenne supérieure à $1800 \mathrm{~m}$. Cela suffirait à en faire des torrents vigoureux, mais à l'influence de l'altitude s'ajoutent d'autres facteurs: la plupart de ces cours d'eau qui dévalent le versant nord de la chaîne axiale des Pyrénées ont une orientation SE-NW ou SW-NE; malgré une régression paradoxale de la pluviosité dans le sens N-S, Couflens reçoit encore

3. Cartes IGN au $\mathbf{1} / \mathbf{2 0 . 0 0 0}$, coupures Aulus-les-Bains $\mathrm{n}^{\circ} \mathbf{1}, 2,5,6$ et SaintGirons $\mathrm{n}^{\circ} 5,6$. Cadastres anciens de 1838-39 pour les trois communes; cadastres rénovés de Couflens (1956), Ustou (1957) et Seix (1960).

4. Cassini: $n^{\circ} 40(1775-1781)$.

5. L'informateur principal et irremplaçable par sa connaissance du milieu a été mon collègue Roger BarraU, maire de Seix, conseiller général de l'Ariège. M. SAUBENS, infatigable chercheur et l'un des meilleurs connaisseurs du Couserans, m'a également donné par écrit de très utiles précisions. J'ai parcouru moi-même plusieurs fois l'aire des hoques en juillet et août 1974.

Les documents anciens utilisés sont les terriers de Seix de 1519 (AD 09, $136 \mathrm{E}$ suppl. $2^{5}$ ) et de $1672\left(\mathrm{AD} 09,136 \mathrm{E}\right.$ suppl. $2^{4}$ ), celui d'Uston de 1771 (AD 09, E 75) et celui de Couflens de 1778 (AD 09, E 38). 
$1000 \mathrm{~mm}$ d'eau par an et Salau $1050 \mathrm{~mm}^{6}$; la forte nivosité en amont permet d'observer sur les hoques comme sur la plupart des torrents alpestres et pyrénéens une variation du module: le régime nival de montagne donne à celles de Couflens un débit très bas en hiver et énorme en juin, alors que le régime nival «de plaine», aidé par une pluviosité plus grande, provoque des maxima en avirl et mai ${ }^{7}$. Comme presque tous les ruisseaux de montagne, les hoques présentent donc un cours très contrasté, alternant minceur $d$ 'un écoulement saisonnièrement appauvri et ravinement brutal des «eaux sauvages» ${ }^{8}$.

La géologie finit d'en expliquer le caractère torrentiel. Si «la rivière de Saint-Lizier, l'Alet, a été occupée par un puissant glacier de vallée, la vallée du Salat n'a jamais été englacée en aval de Salau» ${ }^{9}$. Ces remarques de M. Chevalier sur le «modelé purement fluviatile» de l'aire des hoques reposent sur la structure géologique du bassin du Haut-Salat. Sauf une suele exception (L'hoque de Goueillou à Ustou) ${ }^{10}$, toutes les hoques proviennent $\mathrm{du}$ ravinement intense des schistes carburés, ardoisiers ou satinés de l'étage Silurien, flanqués sur leur périphérie des couches calcaires du sente des sommets de plus de 2300 m, du Cap de Ruhos au Tuc de Quer Ner, c'est le granite de la zone axiale qui tranche la limite orientale. Les schites cristallins des massifs nord-pyrénéens bornent l'aire dans la commune de Sentenac-d'Oust, alors que les calcaires secondaires métamorphiques constituent le flanc droit de la vallée de l'Alet et s'avancent, au $\mathrm{NW}$, dans dans le synclinorium intra-pyrénéen jusqu'au sommet de la Souleille $d^{\prime}$ Esbints ${ }^{11}$. Les granites sont à peine érodés par les «recs», tandis que les «gouttes» correspondent à l'hydrologie karstique du nord de l'Alet.

Les hoques apparaissent donc comme caractéristiques des schistes siluriens fortement ravinéssous l'influence du régime des précipitations renforcé par l'altitude, l'absence de modelé glaciaire, l'étroitesse même des bassins de réception de ces menus affluents du Salat et l'extrême brièveté de leur profil. Ce sont des torrents, mais dans une aire dont la géologie et la tectonique ont fait un isolat, au moins géographique.

6. Gaussen, H.: La flore des montagnes pyrénéennes dans ses rapports avec le climat quaternaire (Congrès International de Géographie, 1931, 2 (2), 745-750) et Chevalier, M.: Notes sur la morphologie glaciaire du Haut-Salat, R.G.P.S.-O. t. 22 (1951), p. 213.

7. Parde, M.: Fleuves et rivières, Paris, A. Colin, 1933, pp. 19, 27, 98.

8. Parde, M.: ibid. p. 57.

9. Chevalier, M.: op. cit., p. 209 et Le relief glaciaire des Pyrénées du Couserans, R. G.P.S.-O., t. 25 (1954), p. 190.

10. Ustou, Plan cadastral, A 4.

11. Chevalier, M.: R.G.P.S.-0, t. 25 (1954), p. 117 (Coupes). 
TRANGOIS BABY

\section{Definition}

Torrent mais aussi, par une métonymie habituelle, ravin dans lequel elle coule, une hoque a le statut linguistique d'un véritable appellatif générique de cours $d^{\prime}$ eau ${ }^{12}$.

Au critère habituellement retenu de la multiplicité des occurrences dans un milieu géographique ou linguistique donné, il semble que l'on puisse ajouter sept autres conditions nécessaires à l'identification d'un appellatif générique:

I-Quantité: Avec 137 mentions pour $260 \mathrm{~km}^{2}$, hoque répond au critère quantitatif avec une densité très suffisante.

2 - Elément d'un syntagme: Le mot peut entrer dans un syntagme nominal ou adjectival.

- Syntagme nominal: généralement associé à un lieudit, comme dans Loque d'Estours, de Fach, de Saillens, de Madrits, las oquas de Crabilhera à Seix ${ }^{13}$, Loque d'Izet, des Trauquets, de Trabes, de la Souleye, de Ouergrand, des Hustes, del Bouch, de Palots, de Riumaut, d'Infer à Couflens ${ }^{14}$, et à Ustou Locque d'Elber, $d^{\prime}$ Ardie, de la Souleillotte ${ }^{15}$.

-Souvent associé à un anthroponyme, presque toujours celui d'un propriétaire: ainsi Loque de David à Couflens ${ }^{16}$, Loco de Guillamet à Seix ${ }^{17}$.

-Parfois en chaîne: Locque de la Font de la Turere, Loque du Prat de Bernard Barrau à Seix ${ }^{18}$.

- Syntagme adjectival (avec ou sans un second élément) comme dans Loque granne, Loque rouch des Pieulos à Couflens en $1778{ }^{19}$.

3 - L'emploi absolu apparaît généralement dans les confronts de lieux-dits: ainsi à Seix en 1585 «terra en Sausez confronta dam loquo» et en 1672 «terre au Sarrat de Broué confronte avec loque» ${ }^{20}$. Le terrier de Couflens de 1778 permet de relever vingt-deux emplois de ce genre ${ }^{21}$, mais l'exemple majeur nous est donné à Ustou, où hoque désigne le cours

12. Par exemple, pour les rapports RĪPPĀRIA/rivière et GOUTTE/vallée, voir la carte 787 de l'ÁLG. III au points 780 . 790 et 090 NE.

13. Seix, Terrier de 1672 , f. $30+$ v., 359 r., 441 r.; terrier de 1519 , f. 370 bis vi., 350 r. et 353 r. Couflens, terrier de 1778, f. 93 v., 94 v., 102 r., 106 v., 138 v., 7 r., 7 v., 34 r., 48 r., 74 v. Terrier d'Ustou (1771), f. 49 v., 42 v., 191 r.

16. Couflens $(1778)$, f. 57 v.

17. Seix $(1672)$, f. $366 \mathrm{r}$.

18. Seix (1519), f. 197 r.; Seix (1672), f. 64 v.

19. Couflens (1778), f. 34 r.. 94 r.

20. Seix (1585), f. 74 r.; Seix (1672), f. 244 v. et 252 r.

21. Couflens $(1778)$, f. 32 v., 34 v., 46 r., 75 r., 76 v., 83 v., 96 v., 97 r., 97 v.. 106 v., 110 r., 119 r. 120 v., 135 v. 
supérieur de l'Alet en amont de Saint-Lizier avec des lieux-dits comme «au cap de locque», «au pont d'hoques», et «la chapelle du Pont d'Hoques» 22 .

4-Existence $d^{\prime} u n$ pluriel: elle justifie un emploi pleinement conscient du mot; c'est le cas pour La Tire de las Hoques à Ustou en $1771^{23}$ et pour Las Oques de Crabilhera à Seis dès $1519^{24}$.

5 - Existence d'un diminutif ou d'un augmen`atif: On ne relève ici quue le diminutif aikilòt qui reste encore dans I'usage courant à Seix pour désigner un tout petit torrent ${ }^{25}$, mais dont les documents écrits ne livrent qu'une attestation à Ustou avec L'ouquillot de las Rives ${ }^{26}$.

6 - Double:s tautologiques: La multiplication de l'emploi absolu tend à rendre le terme arbitraire, d'où les redondances, d'ailleurs biunivoques, de Loquo de Regolo à Seix dès 1588, Loquo de laigo en 1594, le rieu de $l^{\prime}$ oco en 1672, et à Ustou Le ruisseau de locque en $1771^{27}$.

7 -Place relativement stable dans la hiérarchie des cours d'eau: L'existence d'un diminutif formé par suffixation place hoque à l'avantdernier barreau de l'échelle hydrologique et hydronymique: la hiérarchie apparaît clairement a Seix en 1672, où un pré situé «à la Ruere» (=le Salat) confronte avec le Rieu de Ledrouch et Loque de Moneu, tandis que loque de Crabingues apparaît comme un affluent du Rieu Majou qui se jette dans le Salat ${ }^{28}$. Le système fonctionne surtout par différenciation/opposition, en particulier par rapport à un cours d'eau de débit supérieur, mais aussi par rapport à un autre type de ruisseau de même profil: ainsi s'explique dans la vallée d'Ustou la concurrence Hoque-Goutte pour désigner des ruisselets temporaires, comme des gouttes, mais ravinés, comme des hoques, dans toute la zone où les laves torrenticlles ont partiellement meublé l'auge de l'ancienne vallée glaciaire, créant ainsi un type de relief composite soumis à une érosion très variable.

La confusion intervient parfois en fonction de la priorité statistique: en paysage d'hoques, une authentique goutte au mince module, la gota de la Fogarosa de 1519 devient L'hoque de Faugarech en 1838, isolée qu'elle était parmi vingt-six hoques affluentes du Salat sur les cinq kilo-

22. Ustou (1771), f. 155 v., 154 r. et AD 09, G 303, p. 220 (1776).

23. Ustou (1771), f. 59 r. et Cadastre, E 1.

24. Seix $(1519)$, f. 350 r. el 353 r.

25. Informateur: M. Roger BARraU.

26. Ustou, Cadastre (1838-39), E 4.

27. Seix (1519), f. 181 v. et 1.82 r., 384 v.; Seix (1672), f. 441 r.; Ustou (1771), f. 230. r.

28. Seix (1672), f. 393 r. et 177 v.

29. Seix (1519), f. 385 v.; Cadastre C 3. 
mètres qui séparent Couflens du Pont-de-la-Taule ${ }^{29}$. L'attraction est alors si forte qu'elle saccage la hiérarchie ancienne et que le riu de Madritz de 1519 es déjà devenu loquo de Madritz en $1594^{30}$.

Inversement, au contact des calcaires dolomitiques de l'avancée nordouest du synclinorium intra-pyrénéen, en pleine zone de gouttes, une hoque de 1672 s'appelle aupourd'hui la Goutte du Rouquet ${ }^{31}$.

8 - Capacité de topogénèse: Quand il s'agit de désigner les terrains au voisinage des cours d'eau, l'appellatif générique l'emporte presque toujours sur l'appelatif occasionnel. Cela est constant en zone de montagne, notamment dans le couserans, mosaïque de «petits pays» constitués en unités géographiques, politiques et humaines: chaque alvéole de ce «compartimentage humain» ${ }^{32}$ y a toujours pour axe une rivière dont le nom particulier est évincé au profit du générique dans la dénomination des lieux=dits: ainsi dans la vallée de $1^{\prime}$ Artillac trouve=t=on a la Ribera en $1326^{35}$ et a la Ribere dans la vallée de Bethmale en $1669^{36}$. C'est probablement l'un des critères les plus sûr de l'appellatif.

Hoque $n^{\prime} y$ manque pas, en emploi absolu ou en syntagme plus complexe: explicitement à Couflens en 1778 «au quartier de loque de Farlègue», plus couramment «une terre à locque det Saut», «un pred a loque de Fusté», «une borde et un pred a loque $d^{\prime}$ Aujout» ${ }^{37}$. De même à Seix en 1621 «un ichart de tres pards l'une appellade Locque de Roigole» et en 1672 «une terre a loque des Agouaillous» et une autre «au camp de loque» ${ }^{38}$. Employé absolument, l'appellatif domine, on l'a dit, la haute vallée de l'Alet qu'il désigne constamment en 1771 à Ustou à travers des lieux-dits comme «au cap de locque», «au cot de locque» «a loque» et aujourd'hui encore avec «le pont d'Hoques» et «la chapelle du Pont d'Hoques» ${ }^{39}$.

Ces critères sont applicables aux autres appellatifs génériques des cours d'eau de l'Ariège, encore vivants comme riu, goutte, rec, agal, ou en voie de lexicalisation comme mairal, barat, galage, rejole, etc... ${ }^{40}$.

30. Seix (1519), f. 62 r. (1594), f. 374 v.

31. Seix (1672), f. 441 r.; Cadastre E 3.

32. Segur, J.: Via Domitia, 3, p. 46.

33. AD 31, H Malte, Gabre 6, Chasteau Nouvel, 1 (1326).

34. AD 09, 5 J 2 (1668).

35. AD 31, B, Eaux et Forêts, Comminges, 06 (1552).

36. AD 31, B, Eaux et Forêts, Comminges, 03 (1669).

37. Couflens $(1778)$, f. 10 r., 23 r., 41 r., 130 v.

38. Seix (1519), f. 181 bis v.; Seix (1672), f. 125 v., 317 r.

39. Ustou (1771), ef. note 22 et f. $163 \mathrm{r}$.

10. Cf. Baby, F.: Petite hydronymie ariégeoise (Bulletin de la Société Ariégeoise des Sciences, Lettres et Arts, 30 (1975. 


\section{ETyMologie}

1 - La pronunciation locale gko et la graphie oco/oque dans les documents anciens permettent $d^{\prime}$ écarter un $H$ initial qui semble avoir été appelé moins par I'esquisse d'une Hiatustilgung après l'article que par une paronomase avec hoc $(=\mathrm{feu})$ dans une dialectique populaire de l'eau et du feu simplement résolue par une opposition de genre. Hoque est la forme française, cadastrale, officielle, calquée du gascon.

2 - Phonétique et sémantique permettent d'écarter un étymon gaulois OLCA (=terre labourable, enclos) origine probable de L'Ouche/Louche, nom de huit lieux-dits du Loiret, de la Nièvre, du Cher et de l'Indre ${ }^{41}$, ainsi que son dérivé *OLCIA (ou OSCA avec développement en -ya) qui semble représenté dans Ochiaz (Ain, 1344) ${ }^{42}$. Bien plus une étymologie par le celtique d'un hydronyme pyrénéen est tenue, depuis DAUZAT, comme hautement improbable.

De même le traitement en - yK- du groupe - SK - en gascon couserannais ne permet pas de recourir à la base pré-latine OSK - qui semble hydronymique si l'on s'en rapporte au nom de $L^{\prime} O u c h e$, rivière de la Côte-d'Or (Oscara, 584), et d'Oches, localité de la Meuse à la source d'une rivière du même nom (Oschera, 1049; Oscara, XII ${ }^{\circ}$ s. $)^{43}$.

3 - HOQUE paraît directement issu du latin OC̆CA (=herse) dont la voyelle tonique s'est maintenue ouverte, alors que le diminutif ŎCC-IO(U) L-O'TTUM (?) devenait akilòt par suite du traitement de l'initiale atone qui a fait passer OCCASIŌNEM à akazyu et OCCUPĀRE à akupa dans le courante du $X^{\circ}$ siècle ${ }^{44}$. Vieux mot cité par Caton, supplanté par crates en latin impérial, puis par hirpex comme souche romane prioritaire, OCCA est bien représenté en vieux gallois, breton, vieux haut allemand, vieil anglais, lituanien et lette: son origine indo-européenne est sans doute à rapprocher de celle du latin ācer et du grec oxina (=herse, chez Hesychius). La géminée - CC - autant que Vegete («Crates quae scca vocatur a vulgo») attestent un emploi populaire et rural ${ }^{45}$.

41. Soyer, J.: Recherches sur l'origine et la format on des noms de lieux du département du Loiret, $\S$ 127. DU CANGE: Glossarium mediae et infimae latinitatis, t. 4 , c. 707 y voit des continuateurs de OCCA.

42. Philipon: Dictionnaire topographique du département de l'Ain, Paris, Imprimerie Nationale, 1911 et Dauzat-Rostanng: Dictionnaire des noms de lieux de France, Paris, Larousse, 1963.

43. Davzat-Rostaing: Op. cil.

44. RonJat: Grammaire $(h)$ istorique des parlers provençaux modernes (Monipellier, Société des Langues Romanes), § 171.

45. Ernout-Meillets Dictionnaire étymologique de la langue latine, Paris, 1959, p. 456; HASe, C. B.: Thesaurus graecae linguae, Paris, Didot, t. V, 2.047 a; Freund, G:. Grand dictionnaire de la langue latine, t. II, p. 625 a. 
Mais si le verbe OCCARE a des continuateurs dans les dialectes fran. çais de l'est (ocaí, à Montbéliard) et en castillan (ocar), le simple OCCA offre peu de représentants dans les langues romanes: avec le sens de herse on le trouve seulement en trentin (okka), en portugais ( $\overline{0} \bullet 0$ ) et en galicien (occo) ${ }^{46}$. Le provençal connaît oco au sens de «carline», variété de chardons des terrains sees ${ }^{47}$. Nulle part le mot $\mathbf{n}^{\prime}$ apparaît au sens de ruisseau. Cependant le castillan hueco (= spongieux, mouillé) a permis à M. Joan Coromines de ruiner définitivement une étymologie par VŎCUUS avancée par J. CoRnu, et, à partir du sens de OCCARE chez Varron et Cicerón, de proposer l'analyse sémantique: herser $\rightarrow$ rendre humide $\rightarrow$ être humide, être creux ${ }^{48}$

Peut-on imaginer un emprunt $d u$ gascon couserannais jko au castillan hueco? Certes les rapports transpyrénéens de la récrion du Haut-Salat avec le Haut-Paillars sont anciens et continus: dès le XIII ${ }^{\circ}$ sićcle - et au moins jusqu'au XVII - la commanderie de Saint-jean-de-Jérusalem de Salau a des possessions à Isil, Isavarre ei Alós ${ }^{49}$; les lies et passeries unissent encore en 1704 les éleveurs de Seix et ceux de Salardu ${ }^{50}$; les ports d'Aula et de Salau ont toujours été des «pénétrantes» économiques el pourraient avoir permis des emprunts linguistiques.

Mais, pour ne rien dire des difficultés de conversion en substantif gascon d'un déverbal castillan, il faut noter que les dérivés ibéro-romans OCCCĀRE sont relevés seulement en des points discontinus (portugais, galicien, Burgos, Santander), sans le moindre contact avec l'aire compacte des hoques gasconnes. Le catalan de la haute vallée de la Yoguera Pallaresa ne connaît pour torrent que le mot barranco et ignore totalement les produits de OCCA, OCCĀRE, OCC ÁTUM ${ }^{51}$ : une moindre nivosité, un régime plus modeste des précipitations, le modelé beaucoup moins raviné de ce versant sud de la chaine axiale expliquent amplement cette marque négative. Il n'y a done pas eu d'emprunt.

De plus la filiation sémantique proposée par Joan Cokomnes (hersage $\rightarrow$ humidité $\rightarrow$ creusement) semble ici sans objet. Le gascon connaît pourtant trente-neuf mots vivants pour désigner les terrains meubles, légers, humides et marécageux, mais aucun ne provient de OCCARE ${ }^{52}$.

46. Wantblrg, W. von: Französisches etymologisches Wörterbuch. VII, 294 b.

17. Mistral: Lou tresor d'ou felibrige, Remondel-Aubin, Aix.

48. Conommas. J.: Diccionario crítico etimológico de la lengua castellana (Berne. Francke; Madrid. Gredos, 1951, t. II, 965 et Anules del Instiluto de Lingïistica Universidad Nacional de Cuyo, Mendoza, I, pp. 137-142 (1942).

19. AD 31, H Malte, Caignac, 53, $n^{\circ} 7$ (1299) el $n^{\circ} 19$ (1604).

50. AD 09, 136 E suppl. (Lettre des jurats de Salardu aux consuls de Seix. 25 Juin 1704).

51. Enquête personnelle dans la vallée d'Isil (juillet 1974) principalement à Alós de Isil.

52. ALG, II. p. 21. 
4-Pour hoque il faut sans doute s'en tenir à l'image immédiate de la herse: hersage $\rightarrow$ creusement, herse $\rightarrow$ ravin. Pour rendre compte des phénomènes d'érosion, ce type de métaphore est spontané sous la plume des géographes: «les vallées secondaires lacèrent les massifs» ${ }^{53}$, «les parois verticales sont profondément griffées par le ravinement» ${ }^{54}$, «un bassin de réception aux flanc très déclives, dénudés, balafrés de rigoles» ${ }^{55}$, et à propos des entailles parallèles des hoques dans le massif du Haut-Salat: «Le versant ouest... est découpé à intervalles presque réguliers par les traits de scie des ravins latéraux» ${ }^{56}$. Les hoques agissent sur le relief comme une herse.

Le provençal oco, Carlina vulgaris des terrains secs, exclut l'eau de sa sémantique; réciproquement les Pyrénées Centrales ne fournissent aucun terme de botanique en rapport avec hoque =-ruisseau : Jean SEguY, Palay, Alibert, l'auraient relevé ${ }^{57}$. Les deux mots s'expliquent pourtant par la même image: les cardes des bractées du chardon jouent dans l'opération du cardage de la laine le même rôle que les dents de la herse dans le hersage d'un terrain; et les hoques du Haut-Couserans s'adaptent d'autant mieux à ce sens métaphorique généralisé que leurs cours sont souvent parallèles et leur nombre $d^{\prime}$ une forte densité dans une aire restreinte.

Cette image n'est qu'un exemple du vocabulaire de l'érosion, populaire et rural.

Si la zoologie connait «l'araignée herseuse», «dont le bout des tarses est garni $d^{\prime}$ une espèce de brosse» ${ }^{58}$, l'hydronymie emprunte encore bien des termes à l'outillage rural traditionnel.

FŬRCA n'est guère utilisé qu'en vertu de l'analogie de la forme mais est abondamment représenté dans les topo-hydronymes de la Romania: l'espagnol horcajo, l'aranais horcall, l'ancien français fourc, l'ancien logoudorien forkillas, le sarde de Sassari furchillos, le fuxéen forcat et le gascon hourquet désignent le confluent de deux cours d'eau ${ }^{59}$. En Ariège on

53. Goron, L.: Aperçu géographique du département de l'Ariège, Toulouse, Julia, 1957, p. 65.

54. Chevalier, M.: La vie humaine dans les Pyrénées ariègeoises, Paris, Géninde Médicis, 1956, p. 75.

55. Parde, M.: Op. cit., p. 58.

56. Chevalier, M.: R.G.P.S-O, t. 25 (1954), p. 100.

57. Seguy, J., 1953: Les noms populaires des plantes dans les Pyrénées centrales, Barcelona, CSIC, 539 a PALAY: Dictionnaire du béarnais et du gascon moderne, Paris, CNRS, 1961. Alibert: Dictionnaire occitan-français d'après les parlers languedociens, Toulouse, IEO, 1965.

58. FEW, FV, 431 citant BEscherelle: Dictionnaire national ou dictionnaire universel de la langue française, Paris, 1845.

59. Meyer-LübcKe, ReW, 3593; Corominas: Dicc. cril. etim... II, 946; Few, III, 891-892; Allibert: 406; Palay: 577. 
jeut relever le ruisseau de Rieufourcand à Bélesta ${ }^{60}$, les deux Etangs Forcats a Auzat ${ }^{61}$, et, pour les hoques elles-mêmes, lo forquat de Madris à Seix en 1519 et Loque dei Fourc à Couflens en $1778^{62}$.

Mais Furca possède aussi une définition fonctionnelle en occitan et catalan (forcat, forcar $=$ charrue) et pleinement résultative en roumain ( /urc $\breve{a}=$ ornière du chariot $)^{6.3}$.

Comme pour le provençal oco, forme et fonction définissent le portugais restello $(<$ RASTELLLUM $)=$ séran pour peigner le chanvre et le $\operatorname{lin}{ }^{64}$.

Du germanique HRASPŌN proviennent l'engadinois raspa, l'occitan raspino, le roumain raspa, le languedocien raspă (= râpe, lime), dont les dérivés traduisent souvent l'érosion provoquée par une boisson forte, tels le transylvanien raspil ( = eau de vie qui râcle le gosier), le quereynois raspet $(=\operatorname{vin}$ aigrelet $)$, et l'image est vivante du Brabant (raspé = piquette) au Médoc (raspade = vin âpre) peut-être appelée par un autre mot raspa (= uva) cité par du Cange ${ }^{65}$. Mais cette érosion concerne l'hydronymie dans le Pays de Vaud avec rapaye (= averse, ondée qui fouille le sol) et en houergue avec raspa (= courant rapide sur des rochers) où, comme pour OCCCA le simple sert à noter à la fois l'outil et le cours d'eau ${ }^{6 .}$. Cette force érosive connote des oronymes ariégeois comme le Tuc de la Raspe, à Seix, au profil très accidenté et La Raspe de Cocaï à-pic et ravin d'une déclivité de $800 \%$ où coule un sous-affluent de l'Alet à Ustou; elle explique également des hydronymes comme la Raspille $(<*$ RASPICULA) affluent droit du Rhône dans le haut Valais roman, et la Raspe, sous-affluent de l'Arac à Boussenac (Ariège) ${ }^{67}$.

De * RASCLĀRE, dénominatif de * RASCULUM, doublet de RĀSTRUM, l'occitan connaît rascle (= herse) et el portugais racha (= râteau), tandis que le très ancien raschia (1176) (= aqua subsidens, locus aquaticus) conserve également un sens hydronymique à travers le picard $r a$ $q u e^{68}$.

60. Bélẹsta: AD 09, $70 \mathrm{E}$ suppl. 2 et 3.

61. Auzat: $1 \mathrm{GN}$, Vicdessos, $\mathrm{n}^{\circ}$ 5-6.

62. Seix (1519), f. 370 bis v.; Couflens (1778), f. 32 v.

63. Cioranescu: Diccionario etimológico rumano. Madrid, Gredos, 1966, p. 347.

64. MRER-LIBRE. REw, 7078.

65. 6n. 707\%; Chaneste der. 691: Alubert, 580; Palay, 835: de; Cangé: Chossurimm.... t. 5, p. 591 .

66. Meyer-Lïbche: Rl, 7077 et Alibert : 580.

67. IGN, Aulus-les-Bains, $\mathrm{n}^{\circ}$ 1. TAGMANin: Toponymie et vie rurale de la région de Miège (Haut Valais roman), Erlenbach-Zürich, Eugen Rentsch, 1946, p. 2. IGN Saint Gitons, $n^{\circ} 8$.

68. Meyer-Lïbke Rew, 7072 et de Cange: Clossarium..., t. 5, pp. 574-590, 


\section{HOQUE}

Hoques Cadastre et I.G.N. O 1519-1778 Gouttes id. $\square$ id.

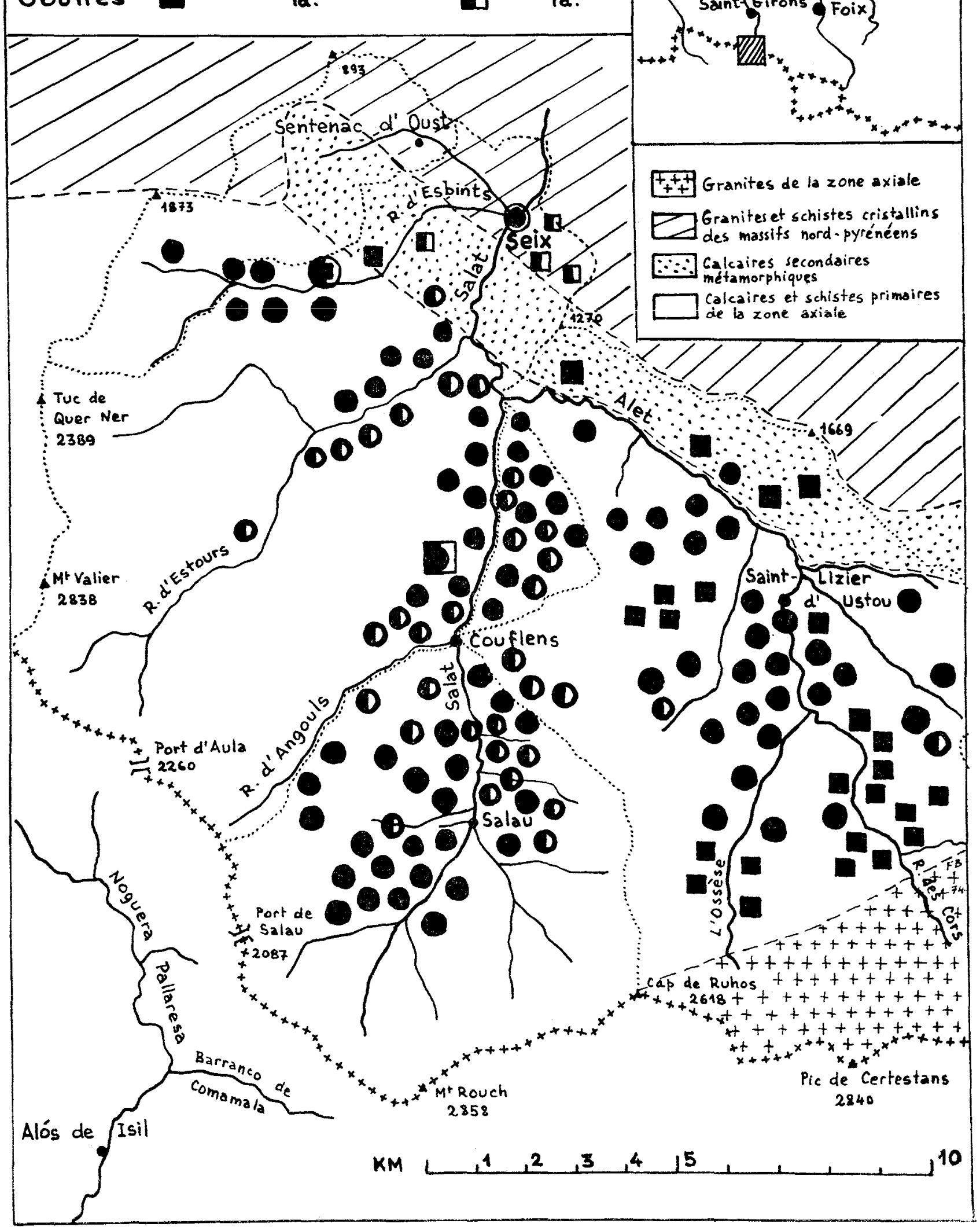


Enfin, si l'on s'en tient pour le languedocien rèk et le gascon arrèk à un croisement du préroman * (KAR) REKKU par le celtique * R ${ }^{*} \mathrm{CA}$ (= sillon) largement attesté et prenant, en aranais, portugais, parlers du Pallars et du Languedoc occidental, le double sens de sillon et de rigole $\mathrm{d}^{\prime}$ écoulement ${ }^{69}$, on ne pourra pas exclure la charrue de cet immense champ sémantique de l'érosion exprimé à travérs les outils les plus simples de la civilisation rurale traditionnelle.

Hoque s'explique done autant par la structure géographique de son aire que par sa situation dans le vocabulaire populaire des instruments aratoires. Comme les autres mots il désigne l'outil et son effet, la fonction et le resultat, le ravinement et le ravin, le ruisseau et son lit.

Terme évidemment typique de la montagne, il survit dans une aire fermée comme ses homologues du Rouergue, du Valais ou du pays de Vaud. Et comme eux il apporte un témoignage supplémentaire du caractère archaïque de ces réduits linguistiques isolés au coeur des hautes Chaînes ${ }^{70}$.

69. Corominas, J.: Tópica hespérica, Madrid, Gredos, 1972, t. II, pp. 110-111, et Diccionario crít. etimol..., III, 1059; Romifs, G.: Le gascon, Pau-Marrimpouey, $2^{\circ}$ éd. 1970, § 63; Dotrin, G.: La langue gauloise, Paris, Klincksieck, 1920, p. 281. 70. Sur l'archaïsme linguistique du Couserans cf. SEgnY, J.: Les noms de plantes, \$ 831 . 